\title{
Projective normality of Weyl group quotients
}

\author{
S.S.Kannan, S.K.Pattanayak \\ Chennai Mathematical Institute, Plot H1, SIPCOT IT Park, \\ Padur Post Office, Siruseri, Tamilnadu - 603103, India. \\ kannan@cmi.ac.in, santosh@cmi.ac.in
}

June 12, 2022

\begin{abstract}
In this note, we prove that for the standard representation $V$ of the Weyl group $W$ of a semi-simple algebraic group of type $A_{n}, B_{n}, C_{n}, D_{n}, F_{4}$ and $G_{2}$ over $\mathbb{C}$, the projective variety $\mathbb{P}\left(V^{m}\right) / W$ is projectively normal with respect to the descent of $\mathcal{O}(1)^{\otimes|W|}$, where $V^{m}$ denote the direct sum of $m$ copies of $V$.
\end{abstract}

Keywords: line bundle, polarizations.

\section{Introduction}

Let $G$ be a semi-simple algebraic group over $\mathbb{C}$ and $W$ be the corresponding Weyl group. Let $V$ be the standard representation of $W$. By Noether's theorem the $\mathbb{C}$-algebra of invariants $\mathbb{C}\left[V^{m}\right]^{W}$ is finitely generated, (see [14]), where $V^{m}$ denote the direct sum of $m$ copies of $V$. So it is an interesting problem to study GIT- quotient varieties $V^{m} / G=\operatorname{Spec}\left(\mathbb{C}\left[V^{m}\right]^{W}\right)$ and $\mathbb{P}\left(V^{m}\right) / W$, (see [12] and [13]). Also, $\forall x \in \mathbb{P}\left(V^{m}\right)$, the isotropy $W_{x}$ acts trivially on the fiber of the line bundle $\mathcal{O}(1)^{\otimes|W|}$ at $x$. Hence, by Proposition (4.2), page 83 of [10], the line bundle $\mathcal{O}(1)^{\otimes|W|}$ descends to the quotient $\mathbb{P}\left(V^{m}\right) / W$, where $\mathcal{O}(1)$ denotes the ample generator of the Picard group of $\mathbb{P}\left(V^{m}\right)$. We denote it by $\mathcal{L}$. On the other hand, $V^{m} / W$ is normal. So, it is a natural question to ask if $\mathbb{P}\left(V^{m}\right) / W$ is projectively normal with respect to the line bundle $\mathcal{L}$. In [9] it is shown that the projective variety $\mathbb{P}\left(V^{m}\right) / W$ is projectively normal with respect to the line bundle $\mathcal{L}$ for $m=1$. In this paper we show that $\mathbb{P}\left(V^{m}\right) / W$ is projectively normal with respect to the line bundle $\mathcal{L}$ for any $m$. At the end of the paper we give a counter example showing that the result does not hold for symmetric groups over a field of positive characteristic. 
The layout of the paper is as follows:

Section 2 consists of preliminary notations and definitions.

Section 3 consists of the main theorem, a corollary and a counter example.

\section{Preliminary notations and definitions}

Let $G$ be a semi-simple algebraic group of rank $n$ over $\mathbb{C}$. Let $T$ be a maximal torus of $G$. Let $N_{G}(T)$ be the normaliser of $T$ in $G$ and let $W=N_{G}(T) / T$ be the Weyl group of $G$ with respect to $T$. Consider the standard representation $V=\operatorname{Lie}(T)$ of $W$. For every integer $m \geq 1$, the group $W$ acts on the algebra $\mathbb{C}\left[V^{m}\right]$ of polynomial functions on the direct sum $V^{m}:=V \oplus \cdots \oplus V$ of $m$ copies of $V$ via the diagonal action

$$
(w f)\left(v_{1}, \cdots, v_{m}\right):=f\left(w^{-1} v_{1}, \cdots, w^{-1} v_{m}\right), f \in \mathbb{C}\left[V^{m}\right], w \in W .
$$

If $m=1$ then the algebra $\mathbb{C}[V]^{W}$ of invariants in one vector variable is generated by $n$ algebraic independent homogeneous invariants $f_{1}, f_{2}, \cdots, f_{n}$ of degrees $d_{1}, d_{2}, \cdots, d_{n}$ respectively such that $\prod_{i=1}^{n} d_{i}=|W|$ by a theorem of Chevalley-Serre-Shephard-Todd (see $[1],[8],[16],[17])$. We will refer to such a system of generators of $\mathbb{C}[V]^{W}$ as a system of basic invariants. Explicit systems of basic invariants are known for each type of irreducible Weyl groups $W$ (see [11]).

Although a system of generators for $\mathbb{C}\left[V^{m}\right]^{W}$ is not given independent of the Weyl group $W$, the classical approach to find a system of generators for $\mathbb{C}\left[V^{m}\right]^{W}$ is by the method of polarization. For type $A_{n}$, by a theorem of Hermann Weyl (see [19]) the algebra $\mathbb{C}\left[V^{m}\right]^{S_{n}}$ is generated by polarizations of the elementary symmetric polynomials. For the Weyl groups of types $B_{n}, C_{n}$ and $G_{2}$, the algebra $\mathbb{C}\left[V^{m}\right]^{W}$ is generated by polarizations of the basic invariants (see page 811 of [18]). However Wallach (see [18]) showed that the polarizations of the basic invariants do not generate $\mathbb{C}\left[V^{m}\right]^{W}$ for type $D_{n}$ and for $m \geq 2$.

Now we will recall the definition of polarizations of a polynomial from page 5 of [19]. Let $f \in \mathbb{C}[V]^{W}$ be a homogeneous polynomial of degree $d$. For $v_{1}, v_{2}, \cdots, v_{m} \in V$ and $t_{1}, t_{2}, \cdots, t_{m}$ are indeterminates, we consider the function $f\left(\sum_{i} t_{i} v_{i}\right)$. Then

$$
f\left(\sum_{i} t_{i} v_{i}\right)=\bigoplus_{\alpha \in\left(\mathbb{Z}^{+}\right)^{m},|\alpha|=d} f_{\alpha}\left(v_{1}, \cdots, v_{m}\right) t^{\alpha},
$$

where the $f_{\alpha} \in \mathbb{C}\left[V^{m}\right]^{W}$ are multihomogeneous of the indicated degree $\alpha$. Here for $\alpha=$ $\left(a_{1}, a_{2}, \cdots, a_{m}\right) \in\left(\mathbb{Z}^{+}\right)^{m}$, we have $t^{\alpha}=t^{a_{1}} \ldots t^{a_{m}}$ and $|\alpha|=a_{1}+\ldots+a_{m}$. We call the polynomials $f_{\alpha}$, the polarizations of $f$.

Polarizations of a polynomial can also be defined in terms of some linear differential operators called the polarization operators. Choosing a basis for $V$ and writing $v_{i}=\left(x_{i 1}, \cdots, x_{i n}\right)$ we define 


$$
D_{i j}=\sum_{k=1}^{n} x_{i k} \frac{\partial}{\partial x_{j k}}
$$

The operators $D_{i j}$ 's are called polarization operators. They commute with the action of $W$ on $\mathbb{C}\left[V^{m}\right]$ and applying successively operators $D_{i j}(i>j)$ to $f \in \mathbb{C}[V]^{W}$ we obtain precisely (up to a constant) the polarizations of $f$ in any number of variables.

Before ending this section we will recall the following.

Let $G$ be a finite group and $V$ be a finite dimensional, faithful representation of $G$ over an algebraically closed field of characteristic not dividing $|G|$. Let $\mathcal{O}(1)$ denote the ample generator of the Picard group of $\mathbb{P}(V)$. Let $\mathcal{L}$ denote the descent of the line bundle $\mathcal{O}(1)^{\otimes|G|}$ to the quotient $\mathbb{P}(V) / G$.

A polarized variety $(X, \mathcal{L})$ where $L$ is a very ample line bundle is said to be projectively normal if its homogeneous coordinate ring $\oplus_{n \in \mathbb{Z}_{\geq 0}}\left(H^{0}\left(X, \mathcal{L}^{\otimes n}\right)\right)$ is integrally closed. For a reference, see exercise 3.18, page 23 of [4].

The polarized variety $(\mathbb{P}(V) / G, \mathcal{L})$ is

$$
\operatorname{Proj}\left(\oplus_{n \in \mathbb{Z}_{\geq 0}}\left(H^{0}\left(\mathbb{P}(V), \mathcal{O}(1)^{\otimes n|G|}\right)\right)^{G}\right)=\operatorname{Proj}\left(\oplus_{n \in \mathbb{Z}_{\geq 0}}\left(\operatorname{Sym} m^{n|G|}\left(V^{*}\right)\right)^{G}\right) .
$$

For a reference, see Theorem 3.14 and page 76 of [12].

Now we will state a combinatorial lemma which can be applied to prove our main theorem.

Let $\underline{d}=\left(d_{1}, d_{2}, \cdots d_{r}\right) \in \mathbb{N}^{r}$ and $N=\left(\prod_{i=1}^{r} d_{i}\right)$. Consider the semigroup

$M_{\underline{d}}=\left\{\left(m_{1}, m_{2}, \cdots m_{r}\right) \in \mathbb{Z}_{\geq 0}^{r}: \sum_{i=1}^{r} m_{i} d_{i} \equiv 0 \bmod N\right\}$ and the set

$S_{\underline{d}}=\left\{\left(m_{1}, m_{2}, \cdots m_{r}\right) \in \mathbb{Z}_{\geq 0}^{r}: \sum_{i=1}^{r} m_{i} d_{i}=N\right\}$.

Lemma 2.1. $M_{\underline{d}}$ is generated by $S_{\underline{d}}$ for $\underline{d} \in \mathbb{N}^{r}$.

Proof. See lemma (2.1) of [9].

\section{Main Theorem}

In this section we will prove our main theorem.

Theorem 3.1. Let $G$ be a semi-simple algebraic group of type $A_{n}, B_{n}, C_{n}, D_{n}, F_{4}$ or $G_{2}$. Let $W$ denote the corresponding Weyl group. Let $V$ be the standard representation of $W$. Then $\mathbb{P}\left(V^{m}\right) / W$ is projectively normal with respect to the line bundle $\mathcal{O}(1)^{\otimes|W|}$. 
Proof. By a theorem of Chevalley-Serre-Shephard-Todd (see [1],[8],[16],[17]), the $\mathbb{C}$ - algebra $\mathbb{C}[V]^{W}=\left(\operatorname{Sym}\left(V^{*}\right)\right)^{W}$ is a polynomial ring $K\left[f_{1}, f_{2}, \cdots, f_{n}\right]$ with each $f_{i}$ is a homogeneous polynomial of degree $d_{i}$ and $\prod_{i=1}^{n} d_{i}=|W|$.

Let $R:=\oplus_{q \geq 0} R_{q}$; where $R_{q}:=\left(S y m^{q|W|} V^{m}\right)^{W}$. First we show that $R$ is generated as a $\mathbb{C}$-algebra by $R_{1}$ by dealing with case by case.

Type $A_{n}, B_{n}, C_{n}$ :

For the diagonal action of the Weyl group on $V^{m}$, in type $A_{n}$ by H. Weyl (see pages 36-39 of [19]) and in type $B_{n}$ and $C_{n}$ by a theorem of Wallach (see page 811 of [18]), the algebra $\mathbb{C}\left[V^{m}\right]^{W}$ is generated by polarizations of the system of basic invariants $f_{1}, f_{2}, \cdots, f_{n}$.

For each $i \in\{1,2, \cdots, n\}$, let $\left\{f_{i j}: j=1,2, \cdots a_{i}\right\}$ denote the polarizations of $f_{i}$ where $a_{i}$ is a positive integer. Since the polarization operators $D_{i j}=\sum_{k=1}^{n} x_{i k} \frac{\partial}{\partial x_{j k}}$ do not change the total degree of the original polynomial, we have

$$
\text { degree of } f_{i j}=\text { degree of } f_{i}=d_{i}, \forall j=1,2, \cdots a_{i} \text {. }
$$

Let us take an invariant polynomial $f \in\left(S y m^{q|W|}\left(V^{m}\right)\right)^{W}$. Since $f_{i j}$ 's generate $\mathbb{C}\left[V^{m}\right]^{W}$ with out loss of generality we can assume $f$ is a monomial of the form $\prod_{i=1}^{n} \prod_{j=1}^{a_{i}} f_{i j}^{m_{i j}}$.

Since $f=\prod_{i=1}^{n} \prod_{j=1}^{a_{i}} f_{i j}^{m_{i j}} \in\left(\operatorname{Sym}^{q|W|}\left(V^{m}\right)\right)^{W}$, we have

$$
\sum_{i=1}^{n} \sum_{j=1}^{a_{i}} m_{i j} d_{i}=q|W|=q\left(\prod_{i=1}^{n} d_{i}\right)
$$

Let $m_{i}=\sum_{j=1}^{a_{i}} m_{i j}$ then we have $\sum_{i=1}^{n} m_{i} d_{i}=q\left(\prod_{i=1}^{n} d_{i}\right)$, and hence $\left(m_{1}, m_{2}, \cdots, m_{n}\right)$ is in the semigroup $M_{\underline{d}}=\left\{\left(m_{1}, m_{2}, \cdots m_{r}\right) \in \mathbb{Z}_{\geq 0}^{r}: \sum_{i=1}^{r} m_{i} d_{i} \equiv 0 \bmod N\right\}$.

By lemma (2.1), the semigroup $M_{\underline{d}}$ is generated by the set $S_{\underline{d}}=\left\{\left(m_{1}, m_{2}, \cdots m_{n}\right) \in \mathbb{Z}_{\geq 0}^{n}\right.$ : $\left.\sum_{i=1}^{r} m_{i} d_{i}=\prod_{i=1}^{n} d_{i}\right\}$. So there exists $\left(m_{1}^{\prime}, m_{2}^{\prime}, \cdots m_{n}^{\prime}\right) \in \mathbb{Z}_{\geq 0}^{n}$ such that for each $i$

$$
m_{i}^{\prime}<m_{i} \text { and } \sum_{i=1}^{r} m_{i}^{\prime} d_{i}=\prod_{i=1}^{n} d_{i}
$$

Again, since $m_{i}^{\prime}<m_{i}=\sum_{j=1}^{a_{i}} m_{i j}$, for each $i$ and $j$ there exists $m_{i j}^{\prime} \leq m_{i j}$ such that

$$
m_{i}^{\prime}=\sum_{j=1}^{a_{i}} m_{i j}^{\prime}
$$

Then $g=\prod_{i=1}^{n} \prod_{j=1}^{a_{i}} f_{i j}^{m_{i j}^{\prime}}$ is $W$-invariant and is in $\left(S y m^{|W|}\left(V^{m}\right)\right)^{W}$.

Let $f^{\prime}=\frac{f}{g}$. Then $f^{\prime} \in\left(S y m^{(q-1)|W|}\left(V^{m}\right)\right)^{W}$ and so by induction on $q, f^{\prime}$ is in the subalgebra generated by $\left(\operatorname{Sym}^{|W|}\left(V^{m}\right)\right)^{W}$. 
Hence $f=g \cdot f^{\prime}$ is in the subalgebra generated by $\left(S y m^{|W|}\left(V^{m}\right)\right)^{W}$.

\section{Type $\mathrm{D}_{\mathrm{n}}$ :}

Before proving the theorem for this case let us recall the action of the Weyl group of type $B_{n}$ and $D_{n}$ on the euclidean space $\mathbb{R}^{n}$. Let $W$ and $W^{\prime}$ denote the Weyl group of type $D_{n}$ and $B_{n}$ respectively. Then $W^{\prime}$ acts on $x=\left(x_{1}, x_{2}, \cdots, x_{n}\right) \in \mathbb{R}^{n}$ by permutation of $x_{1}, x_{2}, \cdots, x_{n}$ and the sign changes $x_{i} \rightarrow-x_{i}$ and the group $W$ acts on $x$ by permuting the coordinates and changes an even number of signs. Then it is clear that the group $W^{\prime}$ is generated by the group $W$ and a reflection $\sigma$ defined by

$$
\sigma\left(x_{1}, x_{2}, \cdots, x_{n-1}, x_{n}\right)=\left(x_{1}, x_{2}, \cdots, x_{n-1},-x_{n}\right) .
$$

From [6] we can take the polynomials

$$
f_{i}=\sum_{k=1}^{n} x_{k}^{2 i}, i=1,2, \cdots, n-1
$$

and

for the basic invariants of $\mathbb{C}[V]^{W}$.

$$
f_{n}=x_{1} \cdot x_{2} \ldots x_{n}
$$

For $\mathbb{C}[V]^{W^{\prime}}$ we can take the basic invariants the polynomials

$$
f_{i}=\sum_{k=1}^{n} x_{k}^{2 i}, i=1,2, \cdots, n-1
$$

and

$$
f_{n}^{\prime}=\sum_{k=1}^{n} x_{k}^{2 n} .
$$

For odd $r \geq 1$, define the operator

$$
P_{r}:=\sum_{k=1}^{n} x_{2 k}^{r} \frac{\partial}{\partial x_{1 k}}
$$

where $x_{1 k}, x_{2 k}$ are standard coordinates of $\mathbb{R}^{2 n}$. The operator $P_{r}$ commutes with the diagonal action of $W$ and $W^{\prime}$ on $\mathbb{C}\left[V^{2}\right]$ and preserves $\mathbb{C}\left[V^{2}\right]^{W}$.

Now by [7] and [17] the algebra $C\left[V^{2}\right]^{W}$ is generated by the polarizations of the basic invariants $f_{1}, f_{2}, \cdots, f_{n}$ and the polynomials

$$
P_{r_{1}} \cdots P_{r_{l}}\left(f_{n}\right) \quad\left(r_{i} \geq 1 \quad \text { odd, } \sum_{i=1}^{l} r_{i} \leq n-l\right) .
$$

Note that the degree of the polynomial $P_{r}\left(f_{n}\right)$ is $n+r-1$ and so the degrees of the polynomials $P_{r_{1}} \cdots P_{r_{l}}\left(f_{n}\right),\left(r_{i} \geq 1\right.$ odd, $\left.\sum_{i=1}^{l} r_{i} \leq n-l\right)$ are

$$
n+\left(r_{1}+r_{2}+\ldots+r_{l}\right)-l \leq 2 n-2 .
$$


So $\mathbb{C}\left[V^{2}\right]^{W}$ is generated by homogeneous polynomials of degree $\leq 2 n-2$.

Now we will prove the theorem for type $D_{n}$ by dealing with two cases.

Case $-1: \mathrm{n}$ is even

In this case note that the degrees of the basic invariants $f_{1}, f_{2}, \cdots, f_{n}$ are all even. So the degrees of the polynomials $P_{r_{1}} \cdots P_{r_{l}}\left(f_{n}\right),\left(r_{i} \geq 1\right.$ odd, $\left.\sum_{i=1}^{l} r_{i} \leq n-l\right)$ are all even. Since the polarizations of the basic invariants have the same degrees as the basic invariants, we conclude that in this case the algebra $\mathbb{C}\left[V^{2}\right]^{W}$ is generated by homogeneous polynomials of even degrees less than or equal to $2 n-2$.

Now for $m>2$, by theorem (3.4) of [7], the algebra $\mathbb{C}\left[V^{m}\right]^{W}$ is generated by the polarizations of the generators of $\mathbb{C}\left[V^{2}\right]^{W}$. Again since the polarization operators do not change the degree of the original polynomial we conclude that the algebra $\mathbb{C}\left[V^{m}\right]^{W}$ is generated by homogeneous polynomials of even degrees same as the degrees of the basic invariants. So in this case we can employ the same proof as in the case of type $A_{n}, B_{n}$ and $C_{n}$.

Case $-2: \mathrm{n}$ is odd

In this case since the degree of the basic invariant $f_{n}$ is odd and $r_{i}$ 's are all odd, we have degrees of all the polynomials $P_{r_{1}} \cdots P_{r_{l}}\left(f_{n}\right),\left(r_{i} \geq 1\right.$ odd, $\left.\sum_{i=1}^{l} r_{i} \leq n-l\right)$ are odd.

Again, since for $m>2$, the algebra $\mathbb{C}\left[V^{m}\right]^{W}$ is generated by the polarizations of the generators of $\mathbb{C}\left[V^{2}\right]^{W}$, among the generators of $\mathbb{C}\left[V^{m}\right]^{W}$ we have some odd degree invariants as well which are not necessarily having the same degrees as the degree of $f_{n}$.

Now, let us take one odd degree invariant $f \in \mathbb{C}\left[V^{m}\right]^{W}$ and write

$$
f=\frac{f-\sigma(f)}{2}+\frac{f+\sigma(f)}{2}
$$

where $\sigma$ is the reflection $\left(x_{1}, x_{2}, \cdots, x_{n-1}, x_{n}\right) \rightarrow\left(x_{1}, x_{2}, \cdots, x_{n-1},-x_{n}\right)$ defined before.

Since the $W$ is a normal subgroup of the Weyl group $W^{\prime}$ of type $B_{n}$ and $W^{\prime}$ is generated by $W$ and $\sigma$, we have

$$
\frac{f+\sigma(f)}{2} \in \mathbb{C}\left[V^{m}\right]^{W^{\prime}}
$$

Again, since $f$ is homogeneous of odd degree, the degree of $\frac{f+\sigma(f)}{2}$ is odd and hence equal to 0 since $\mathbb{C}\left[V^{m}\right]^{W^{\prime}}$ is generated by polarizations of the basic invaraints $f_{1}, f_{2}, \cdots, f_{n-1}, f_{n}^{\prime}$ which are all of even degrees. Hence, for an odd degree invariant $f \in \mathbb{C}\left[V^{m}\right]^{W^{\prime}}$, we have

$$
\sigma(f)=-f .
$$

So for any $W$-invariant polynomials $f$ and $g$ of odd degrees we have $\sigma(f . g)=f g$ and hence we conclude that $f^{2}, g^{2}$ and $f g$ are in $\mathbb{C}\left[V^{m}\right]^{W^{\prime}}$.

Now let us take a typical invariant monomial

$$
f=\left(\prod_{i} \prod_{j=1}^{a_{i}} f_{i j}^{m_{i j}}\right) h_{1}^{l_{1}} h_{2}^{l_{2}} \ldots h_{p}^{l_{p}} \in\left(S y m^{q|W|} V^{m}\right)^{W}
$$


where $g_{i j}$ 's $\in \mathbb{C}\left[V^{m}\right]^{W}$ are the even degree invariants of degrees $d_{1}, d_{2}, \cdots, d_{n-1}$ obtained by taking the polarizations of the even degree generators of $\mathbb{C}\left[V^{2}\right]^{W}$ and $h_{i}$ 's $\in \mathbb{C}\left[V^{m}\right]^{W}$ are the odd degree invariants obtained by taking the polarizations of the odd degree generators of $\mathbb{C}\left[V^{2}\right]^{W}$.

Again since $h_{i}^{2}$ and $h_{i} \cdot h_{j}$ are in $\mathbb{C}\left[V^{m}\right]^{W^{\prime}}$, they are polynomials in $g_{i}$ 's and the polarizations of the even degree basic invariant $f_{n}^{\prime}$. So we may assume that $\sum_{i=1}^{p} l_{i}=0$ or 1 .

Suppose $\sum_{i=1}^{p} l_{i}=1$, then $\mathrm{f}$ is of the form

$$
f=\left(\prod_{i} \prod_{j=1}^{a_{i}} f_{i j}^{m_{i j}}\right) . h \in \mathbb{C}\left[V^{m}\right]^{W}
$$

where $h$ is of odd degree, say $t$. So we have

$$
\sum_{i} \sum_{j=1}^{a_{i}} m_{i j} d_{i}+t=q \cdot|W| .
$$

This is not possible since $d_{i}$ 's are all even and $|W|$ is even. So we conclude that $\sum_{i=1}^{p} l_{i}=0$ and hence $f$ is of the form $g_{1}^{m_{1}} g_{2}^{m_{2}} \ldots g_{r}^{m_{r}}$ where $g_{i}$ 's are all of even degrees less than equal to $2 n$. So in this case we can proceed the proof as in the case of Type $A_{n}, B_{n}$ and $C_{n}$.

\section{$\mathrm{TypeF}_{4}$ and $\mathrm{G}_{2}$ :}

Since the cardinality of the Weyl group of Type $G_{2}$ is $12=2^{2} .3$ and the cardinality of the Weyl group of Type $F_{4}$ is $1152=2^{7} .3^{2}$, by Burnside's $p-q$ theorem ( see page 247 of [15]), they are solvable. Hence the result is true for each case by proposition (1.1) of [9].

Now the proof of the theorem follows from exercise 5.14(d), Chapter II of [4].

We deduce the following Result of Chu-Hu-Kang (see [2]) as a consequence of Theorem 3.1.

Corollary 3.2. Let $G$ be a finite group and $U$ be any finite dimentional representation of $G$ over $\mathbb{C}$. Let $\mathcal{L}$ denote the descent of $\mathcal{O}(1)^{\otimes n !}$. Then $\mathbb{P}(U) / G$ is projectively normal with respect to $\mathcal{L}$.

Proof. Let $G=\left\{g_{1}, g_{2}, \cdots, g_{n}\right\}$ and let $\left\{u_{1}, u_{2}, \cdots, u_{k}\right\}$ be a basis of $U$. Let $V$ be the natural representation of the permutation group $S_{n}$. Let $\left\{x_{1}, x_{2}, \cdots, x_{n}\right\}$ be a basis of $V$; then the set $\left\{x_{11}, \cdots, x_{n 1}, \cdots, x_{1 k}, \cdots, x_{n k}\right\}$ is a basis of $V^{k}$.

Consider the Cayley embedding $G \hookrightarrow S_{n}, g \mapsto\left(g_{j}:=g g_{i}\right)$. Then

$$
\eta: \operatorname{Sym}\left(V^{k}\right) \rightarrow \operatorname{Sym}(U), \quad x_{i l} \mapsto g_{i}\left(u_{l}\right)
$$

is a $G$-equivariant and degree preserving algebra epimorphism. 
Now we will use Noether's original argument (see page 2 of [14]) to show that the restriction map

$$
\tilde{\eta}: \operatorname{Sym}\left(V^{k}\right)^{S_{n}} \rightarrow \operatorname{Sym}(U)^{G}
$$

is surjective. For any $f=f\left(u_{1}, \cdots, u_{k}\right) \in \operatorname{Sym}(U)^{G}$, we define

$$
f^{\prime}:=\frac{1}{n}\left(f\left(x_{11}, x_{12}, \cdots, x_{1 k}\right)+\ldots+f\left(\left(x_{n 1}, x_{n 2}, \cdots, x_{n k}\right)\right) \in \operatorname{Sym}\left(V^{k}\right)^{S_{n}} .\right.
$$

Then we have

$$
\begin{gathered}
\tilde{\eta}\left(f^{\prime}\right)=\frac{1}{n}\left(f\left(g_{1}\left(u_{1}\right), g_{1}\left(u_{2}\right), \cdots, g_{1}\left(u_{k}\right)\right)+\ldots+f\left(g_{n}\left(u_{1}\right), g_{n}\left(u_{2}\right), \cdots, g_{n}\left(u_{k}\right)\right)\right) \\
=\frac{1}{n}\left(g_{1} f\left(u_{1}, u_{2}, \cdots, c_{k}\right)+\ldots g_{n} f\left(u_{1}, u_{2}, \ldots, u_{k}\right)\right)=f
\end{gathered}
$$

Hence, $\tilde{\eta}\left(f^{\prime}\right)=f$ and $\tilde{\eta}$ is surjective. So the corollary follows from theorem (3.1).

\section{A counter example :}

Let $F$ be a field of characteristic $p \neq 2$ and $V$ be the natural representation of the permutation group $G=S_{p^{s}}, s \geq 2$ over $F$. Consider $U=\underbrace{V \oplus V \oplus \ldots \oplus V}_{\left(p^{s}\right) ! \text { copies }}$. Then by therem (4.7) of [3] we have

$$
\left(p^{s}\right) \text { ! copies }
$$

$$
\beta(U, G)=\max \left\{p^{s},\left(p^{s}\right) !\left(p^{s}-1\right)\right\}=\left(p^{s}\right) !\left(p^{s}-1\right),
$$

where $\beta(U, G)$ denote the Noether number, which can be defined as the minimal number $t$, such that the algebra $\operatorname{Sym}\left(U^{*}\right)^{G}$ of invariants can be generated by finitely many elements of degree at most $t$.

So there exists a homogeneous polynomial $f \in\left(\operatorname{Sym}^{d} U^{*}\right)^{G} ; d=\left(p^{s}\right) !\left(p^{s}-1\right)$ which is not in the subalgebra generated by $\left(\operatorname{Sym}^{m} U^{*}\right)^{G} ; m \leq(d-1)$.

Hence, $f \in R_{\left(p^{s}-1\right)}=\left(\operatorname{Sym}^{\left(p^{s}-1\right)|G|} U^{*}\right)^{G}$ but not in the subalgebra generated by $R_{1}=$ $\left(S y m^{|G|} U^{*}\right)^{G}$. Thus, projective normality does not hold in this case.

Remark 1: We couldn't find any reference for the generators of $\mathbb{C}\left[V^{m}\right]$ for type $E_{6}, E_{7}, E_{8}$. We are now working on it. Due to time constraint, we will write it in the future work.

Remark 2: We beleive that from theorem (3.1), we will be able to prove the Projective normality result for any finite dimensional representation of any Weyl group. We are working on this problem. 


\section{References}

[1] C.Chevalley, Invariants of Finite Groups Generated by Reflections, Amer. J. Math. 77(1955), 778-782.

[2] Huah Chu, Shou-Jen Hu, Ming-chang Kang, A note on Projective normality, preprint.

[3] P. Fleischmann, A new degree bound for Vector Invariants of Symmetric Groups, Trans. Amer. Math. Soc. 350 (1998), 1703-1712.

[4] R. Hartshorne, Algebraic Geometry, Graduate Texts in Math., 52, Springer-Verlag, New York-Heidelberg, 197.

[5] J.E. Humphreys, Introduction to Lie algebras and representation theory, Springer, Berlin Heidelberg, 1972.

[6] J.E. Humphreys, Reflection Groups and Coxeter Groups, Cambridge Univ. Press, Cambridge, 1990.

[7] M. Hunziker, Classical invariant theory for finite reflection groups, Transform. Groups 2 (1997) (2), pp. 147-163.

[8] R.Kane, Reflection Groups and Invariant theory, CMS Books in Mathematics, SpringerVerlag, 2001.

[9] S.S. Kannan, S.K. Pattanayak, Pranab Sardar, Projective normality of finite groups quotients. Proc. Amer. Math. Soc. 137 (2009), no. 3, pp. 863-867.

[10] H.Kraft, P.Slodowy and T.A.Springer, Algebraic Transformation Groups and Invariant Theory, Birkhauser, 1989.

[11] M.L. Mehta, Basic set of invariant polynomials for finite reflection groups, Com. in Alg., 16. (5), (1988), 1083-1098.

[12] D.Mumford, J.Fogarty and F.Kirwan, Geometric Invariant theory, Springer-Verlag, 1994.

[13] P.E.Newstead, Introduction to Moduli Problems and Orbit Spaces, TIFR Lecture Notes, 1978.

[14] E.Noether, Der Endlichkeitssatz der Invarianten endlicher Gruppen, Math. Ann. 77, (1916), 89-92.

[15] D.J.S. Robinson, A Course in the Theory of Groups, 2nd ed., Springer-Verlag, 1996.

[16] J.P.Serre, Groupes finis d'automorphisms d'anneaux locaux reguliers, Colloq. d'Alg. Ecole Norm. de Jeunes Filles, Paris (1967), 1-11. 
[17] G. C. Shephard and J. A. Todd, Finite Unitary Reflection Groups, Canadian J. Math. 6 (1954), 274-304.

[18] N.R. Wallach, Invariant differential operators on a reductive Lie algebra and Weyl group representations, J. Amer. Math. Soc. 6 (1993) (4), pp. 779-816.

[19] H. Weyl, The Classical Groups. Their Invariants and Representations, Princeton Univ. Press (1946). 